\title{
Guest editorial: special issue on Real-Time and Network Systems
}

\author{
Enrico Bini ${ }^{1} \cdot$ Claire Pagetti $^{2}$ \\ Published online: 28 March 2019 \\ (c) Springer Science+Business Media, LLC, part of Springer Nature 2019
}

This issue of the journal Real-Time Systems contains extended versions of four selected papers among the ones presented at the 25th International Conference on Real-Time and Network Systems (RTNS 2017), held in Grenoble in October 2017. The scope of the conference covered all aspects of real-time systems and networks. A selection of the papers presented at the conference were invited to submit extended versions for this special issue. These papers were selected based on their quality, relevance and originality.

- The paper entitled "Real-Time Multiprocessor Locks with Nesting: Optimizing the Common Case" is written by Catherine E. Nemitz, Tanya Amert and James H. Anderson. The work proposes an efficient adaptation of real-time nested locking protocol that ensures contention-sensitive non-nested lock requests and incurs low lock/unlock overhead comparable to that of single-resource protocols. The authors have shown the schedulability benefits by presenting a large-scale schedulability study that compares their variants to existing protocols.

- The paper entitled "A Parallel Branch-and-Bound Algorithm to Compute a Tighter Tardiness Bound for Preemptive Global EDF" is written by Mauro Leoncini, Manuela Montangero and Paolo Valente. The paper introduces a parallel exact algorithm, based on branch-and-bound algorithm, to compute an upper bound to tardiness of preemptive Global EDF schedulers. The authors have extensively compared their approach against existing polynomial-time algorithms.

- The paper entitled "Schedulability Analysis of DAG Tasks with Arbitrary Deadlines under Global Fixed-Priority Scheduling" is written by José Fonseca, Geoffrey Nelissen and Vincent Nélis. The work proposes an improved response time analysis for the global fixed-priority (G-FP) scheduling of sporadic real-time

Claire Pagetti

claire.pagetti@onera.fr

Enrico Bini

bini@di.unito.it

1 University of Turin, Turin, Italy

2 ONERA, Toulouse, France 
tasks when each task is modeled by a directed acyclic graph (DAG) of concurrent sub-tasks. Their approach shows significant improvements on the schedulability of DAG tasks for randomly generated task sets compared to state-of-the-art techniques.

- The paper entitled "Hard Real-Time Application Mapping Reconfiguration for NoC-Based Many-Core Systems" is written by Behnaz Pourmohseni, Stefan Wildermann, Michael Glaß and Jürgen Teich. The work introduces a reconfiguration methodology to enable predictable reconfigurations among a given set of pre-computed mappings. A case study on thermal management of many-core systems highlights the benefits of the approach.

We would like to thank the authors that submitted papers to RTNS 2017, and in particular to those who contributed extended versions of their RTNS submissions for inclusion in this journal special issue. We also thank the members of the RTNS 2017 program committee. To guarantee a higher quality of the papers of the special issue, we contacted new reviewers who did not previously review the conference papers. A special thank goes to them for their valuable help. Finally, the General Chairs of RTNS 2017, Dr. Claire Maiza, Dr. Catherine Parent-Vigouroux and Dr. Pascal Raymond (University of Grenoble Alpes, France) deserve a special thank for their enthusiastic involvement in organizing the conference.

Publisher's Note Springer Nature remains neutral with regard to jurisdictional claims in published maps and institutional affiliations.

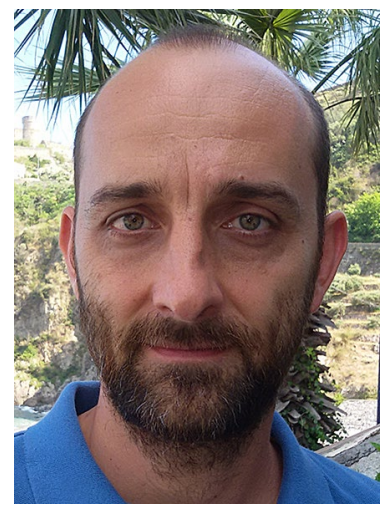

Enrico Bini is Associate Professor at Department of Computer Science, Università di Torino. Until 2016, he was assistant professor at Scuola Superiore Sant'Anna in Pisa. Also, in 2012-14 he was MarieCurie fellow at Lund University, Dept. of Automatic Control. In 2004, he completed the PhD on Real-Time Systems at Scuola Superiore Sant'Anna (recipient of the "Spitali Award" for best $\mathrm{PhD}$ thesis of the whole university). In January 2010 he also completed a Master degree in Mathematics with a thesis on optimal sampling for linear control systems. He has published more than 90 papers ( 4 best-paper awards@RTNS@RTCSA @ICC, 3 most cited papers @ECRTS, 1 most cited @RTSS) on real-time scheduling, operating systems, optimization methods for real-time and control systems, optimal management of distributed and parallel resources. His service to the research community includes the participation in 68 Technical Program Committees (including RTSS (2010, 2014, 2015, 2017, 2018), RTAS (2009, 2010, 2011, 2013, 2015, 2016), EMSOFT (2011, 2016)), the organization of 12 events (including PC co-Chair of RTNS 2017, Local co-Organizer of ESWeek 2018), the review of $13 \mathrm{PhD}$ thesis and about 40 papers/ year, in the above mentioned research areas. 


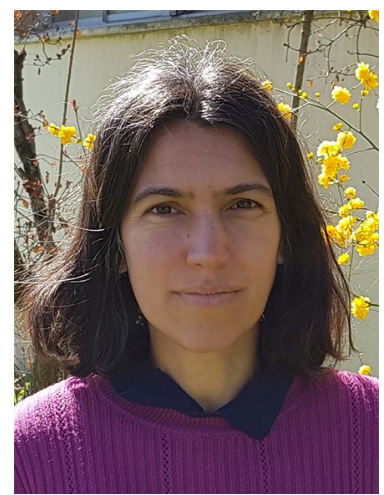

Claire Pagetti is a researcher at ONERA since 2005 and an associate professor at ENSEEIHT since 2007. She defended her habilitation (HDR) in 2015. From Sept 2004 to May 2005, she was a post-doctoral fellow at INRIA. She defended her $\mathrm{PhD}$ in 2004 at the Ecole Centrale de Nantes. Her fields of interest concern the safe implementation of control command avionic applications on avionic platforms. She has contributed to several industrial, European and French projects that lead to several publications, industrial development and a patent. She was responsible of the Torrents cluster and participated to French GDR groups. From September 2016 to August 2017, she was on a sabbatical at TUHH where she worked on WCET-aware compilation of synchronous programs with the WCC compiler. 